\title{
Feature extraction using spectral centroid and mel frequency cepstral coefficient for Quranic accent automatic identification
}

\begin{abstract}
This paper presents the process of Quranic Accent Automatic Identification. Recent feature extraction technique that is used for Quranic verse rule identification/Tajweed include Mel Frequency Cepstral Coefficients (MFCC) which prone to additive noise and may reduce the classification result. Therefore, to improve the performance of MFCC with addition of Spectral Centroid features and is proposed for used in feature extraction of Quranic accents. Through implementing the Spectral Centroid Feature, it complements in improving the accuracy result of identifying the Quranic accents. The pattern classification algorithm here used the dimensional reduced technique from Probabilistic Principal Component Analysis (PPCA) on the features and Gaussian Mixture Model, in purpose to model the effectiveness of both combination of feature extraction. The accuracy of automatic identification for such Quranic Accents are found increasing from $96.9 \%$ to $100 \%$ with the application of SCF.
\end{abstract}

Keyword: Quranic accents automatic identification; Spectral centroid; Feature extraction 